\title{
Artificial Neural Network Modeling for Estimation of Composition of a Ternary Liquid Mixture with its Physical Properties such as Refractive Index, $\mathrm{pH}$ and Conductivity
}

\author{
S. L. Pandharipande \\ Associate Professor, \\ Department of Chemical \\ Engineering, LIT, RTMNU, \\ Nagpur, India.
}

\author{
Anish M. Shah \\ M.Tech sem IV student, \\ Department of Chemical \\ Engineering, LIT, RTMNU, \\ Nagpur, India.
}

\author{
Heena Tabassum \\ B.Tech sem VIII student, \\ Department of Chemical \\ Engineering, LIT, RTMNU \\ Nagpur, India.
}

\begin{abstract}
1. ABSTRACT
The analysis of a ternary mixture can be done by using analytical instruments like TLC, GLC, HPLC, GC etc. which is time consuming \& expensive. In the present work Artificial neural network modeling has been applied to estimate composition of a ternary liquid mixture with its physical properties such as refractive index, $\mathrm{pH} \&$ conductivity. The work is extended in developing ANN model for estimation of composition of a known ternary mixture for the experimentally determined physical properties, refractive index, $\mathrm{pH} \&$ conductivity. Samples having known compositions of a ternary liquid mixture, acetic acid-waterethanol have been prepared \& analysed for the physical properties, refractive index, $\mathrm{pH} \&$ conductivity. ANN models $1 \& 2$ with different topologies have been developed based on the generated data. The predicted \& the actual values using ANN models $1 \& 2$ have been compared based on the \% relative error. The novel feature of this work has been the development of ANN model 1 with the accuracy of prediction between $0-3 \%$ for output parameter, mole $\%$ water \& $0-5 \%$ for output parameter, mole $\%$ acetic acid for training data set $\&$ ANN model 1 having accuracy level of $0-10 \%$ for output parameter, mole $\%$ water \& $0-3 \%$ for output parameter, mole $\%$ acetic acid for test data set.
\end{abstract}

\section{Keywords}

Artificial Neural Network, refractive index, $\mathrm{pH}$, conductivity.

\subsection{INTRODUCTION}

Refractive index, $\mathrm{pH} \&$ conductivity measurements are useful industrially for common substances which include oils, waxes, sugar syrups etc. An exhaustive literature survey reveals that there is data available on physical properties of binary \& some ternary systems, however very few correlations have been reported. ${ }^{[1][2]}$

In the present work known composition of a ternary liquid mixture, acetic acid-water-ethanol has been used for which the refractive index, $\mathrm{pH} \&$ conductivity have been determined. The physical properties, refractive indices, $\mathrm{pH} \&$ conductivity of a ternary liquid mixture is dependent upon its composition. Artificial neural network modeling has been applied to estimate composition of a ternary liquid mixture with its physical properties such as refractive index, $\mathrm{pH} \&$ conductivity.

\subsection{LITERATURE SURVEY Artificial Neural Network}

An Artificial Neural Network (ANN) is an information processing paradigm that is inspired by the way the biological nervous system, such as brain processes information. It is composed of large number of highly interconnected processing elements (neurons) working in unison to solve specific problem. ${ }^{[3]}$

The most common for chemical engineering application is Multi Layer Perception (MLP), which is a feed forward neural network. It consists of input \& output layers with 2-3 hidden layers in between. The nodes between successive layers are connected with weights. The outputs from the input layer are fed to hidden layer units, which in turn, feed their outputs to the next hidden nodes. The hidden node passes the net activation through a non-linear transformation of a linear function, such as the logistic sigmoidal or hyperbolic tangent to compute their outputs. For the training of such a MLP error back propagation algorithm suggested by Rumelhart ${ }^{[4]}$ is popular. This is based on a nonlinear version of the WindroHoff rule known as Generalized Delta Rule (GDR).the schematic of the MLP network developed for the with hidden layer and neurons is shown in fig 1 .

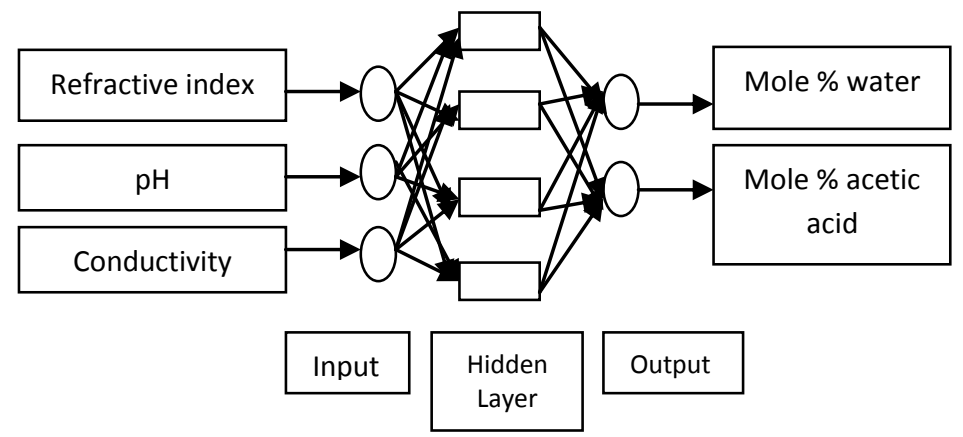

Figure 1: Simple feed forward neural network

There are many applications of ANN reported that include, an approach to fault diagnosis in chemical processes ${ }^{[5]}$, fault diagnosis in complex chemical plants ${ }^{[6]}$, incipient fault diagnosis of chemical process ${ }^{[7]}$, leak detection in liquefied gas pipeline ${ }^{[8],[9]}$, for estimation of mass transfer coefficient for fast fluidized bed solids ${ }^{[10]}$, modeling of distillation 
column $^{[11]}$, detergent formulation ${ }^{[12]}$, modeling of unsteady heat conduction in semi infinite solid ${ }^{[13]}$, prediction of mass transfer coefficient in downflow jet loop reactor ${ }^{[14]}$, modeling of packed column ${ }^{[15]}$, modeling steam table ${ }^{[16]}$, modeling Gurney Lurie \& Heisler charts ${ }^{[17]} \&$ prediction of simple physical properties of mixed solvent system by artificial neural network ${ }^{[18]}$.

\subsection{METHODOLOGY}

The methodology adopted for the present work is given below; the composition of the given ternary mixture can be defined by mentioning mole $\%$ of any two components of the mixture. The ternary mixture having known compositions of acetic acid, water and ethanol have been prepared. The refractive index, $\mathrm{pH} \&$ conductivity of each sample have been determined. ANN model with different topologies have been developed using elite-ANN ${ }^{\circledR[19]}$. The models developed in the present work correlates the composition of the ternary mixture expressed as mole \% of water and acetic acid. The physical properties such as refractive index $\mathrm{pH} \&$ conductivity, referred in the present work as input parameters \& the composition of the ternary mixture as output or dependent parameters.

1.3.1 ANN approach in modeling of estimation of composition of the ternary liquid mixture with the physical properties such as refractive index, $p H \&$ conductivity

The accuracy of the ANN model is dependent upon the number of hidden layers \& number of neurons in each hidden layer. The topology of the ANN models $1 \& 2$ developed in the present work is given in table 1 .

The experimental data set of 66 points is divided into two parts, training \& test data sets having $57 \& 9$ data points respectively.

Table 1: Neural Network Topology
Figure 2: Graph showing the relation between actual and predicted values for ANN model $1 \& 2$ for training data set for prediction of mole \% water

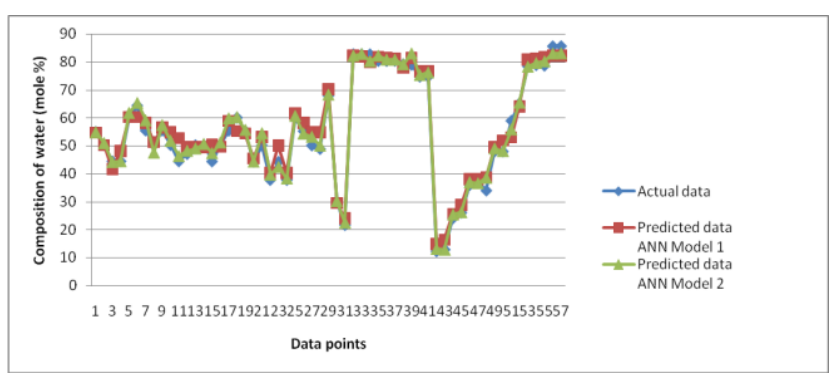

Figure 3: Graph showing the relation between actual and predicted values for ANN model $1 \& 2$ for training data set for prediction of mole \% acetic acid

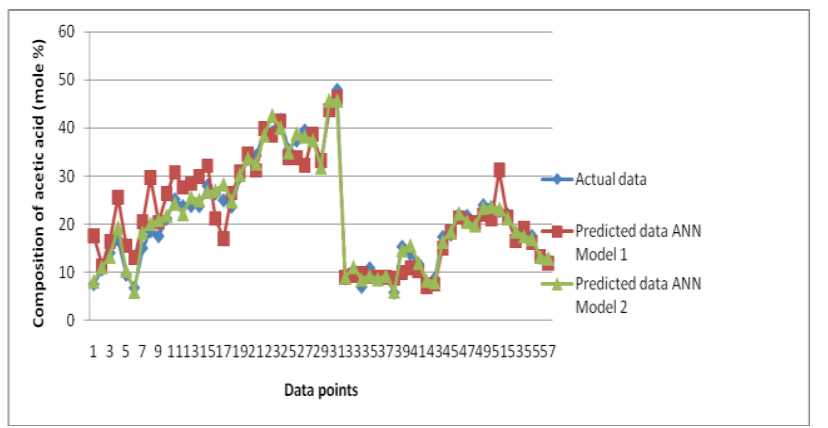

Similarly, a graph is plotted between the actual and predicted values of output parameters namely mole $\%$ water \& mole $\%$ acetic acid respectively for test data set for ANN model $1 \& 2$ as shown in fig. $4 \& 5$ respectively.

\begin{tabular}{|c|c|c|c|c|c|c|c|}
\hline & \multicolumn{5}{|c|}{ No. of neurons } & \multicolumn{2}{|c|}{ Data points } \\
\hline $\begin{array}{l}\text { Type } \\
\text { of } \\
\text { Model }\end{array}$ & Input layer & $1^{\text {st }}$ Hidden layer & $\begin{array}{c}2^{\text {nd }} \text { Hidden } \\
\text { layer }\end{array}$ & $3^{\text {rd }}$ Hidden layer & Output layer & Training & Testing \\
\hline $\begin{array}{c}\text { ANN } \\
\text { Model } \\
1 \\
\end{array}$ & 3 & 0 & 5 & 5 & 2 & 57 & 9 \\
\hline $\begin{array}{c}\text { ANN } \\
\text { Model } \\
2\end{array}$ & 3 & 0 & 10 & 10 & 2 & 57 & 9 \\
\hline
\end{tabular}

The developed models have been used for the estimation of the output parameters for both the training \& test data sets. Comparison has been carried out between actual and predicted values of output parameters. Comparison has also been carried out between predicted values among ANN model 1 and model 2.

\subsubsection{Results and Discussion:}

A graph is plotted between the actual and predicted values of output parameters, mole $\%$ water \& mole $\%$ acetic acid respectively for training data set for ANN model $1 \& 2$ as shown in figures $2 \& 3$ respectively. It can be said that the actual $\&$ predicted values are close to each other. 
Fig. 4: Graph showing the relation between actual and predicted values for ANN model $1 \& 2$ for test data set

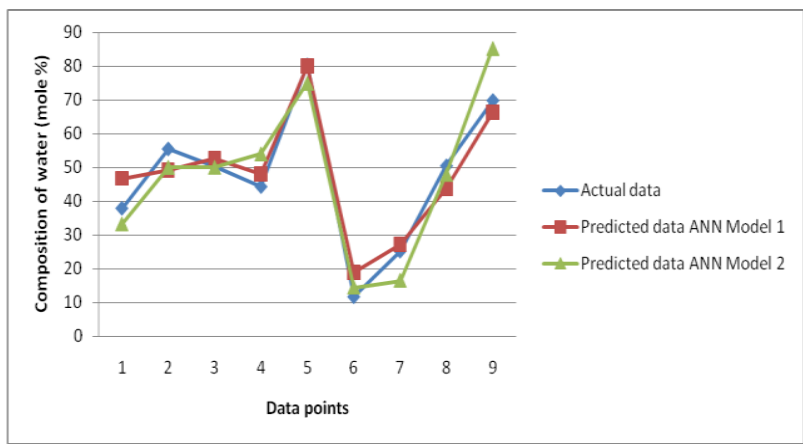

Figure 5: Graph showing the relation between actual and predicted values for ANN model 1 \& 2 for test data set.

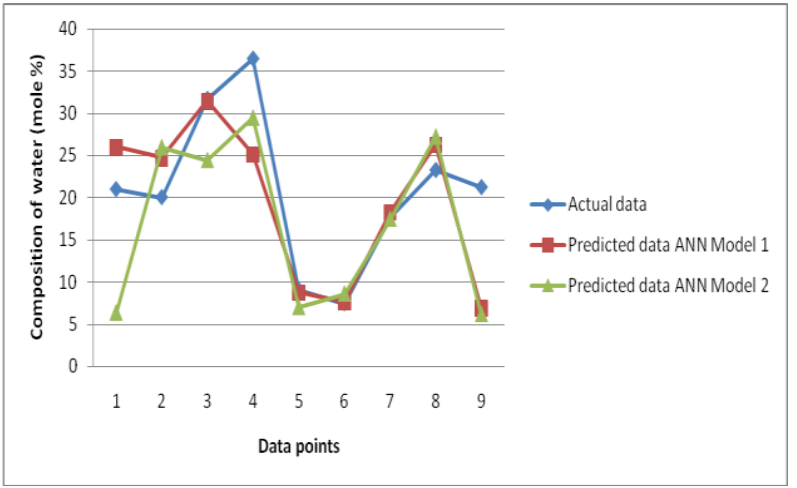

\subsubsection{Comparison of the accuracy of the Model 1 and Model 2 training data set}

The accuracy of the ANN models developed has been further studied by calculating the relative error for all the data points of the output parameters. Fig no.6 \& 7 show the graphs plotted between the \% relative error for the output parameters, composition of mixture expressed as mole $\%$ water and composition of mixture expressed as mole \% acetic acid , for ANN model $1 \&$ ANN model 2 respectively. It is seen that there are deviations of the percentage relative errors from the mean path of the ANN model $1 \& 2$. The range of relative errors for the output parameter, composition of mixture expressed as mole \% water, for ANN model 1 \& ANN model 2 is $0-10 \% \& 0-3 \%$ respectively. Similarly the range for the second output parameter, composition of mixture expressed as mole $\%$ acetic acid, for ANN model $1 \&$ ANN model 2 is $0-20 \%$ \& $0-1 \%$ respectively. As the \% relative error for ANN model 2 is lower for both the output parameters than estimated by ANN model 1, hence it can be inferred that the ANN model 2 is superior to the ANN model 1.

$\%$ Relative error $=\left\{\frac{\text { Actual data }- \text { Predicted data }}{\text { Actual data }}\right\} 100$
Figure 6: Comparison of the relative error for estimation of mole \% of water for ANN model $1 \& 2$ for training data set

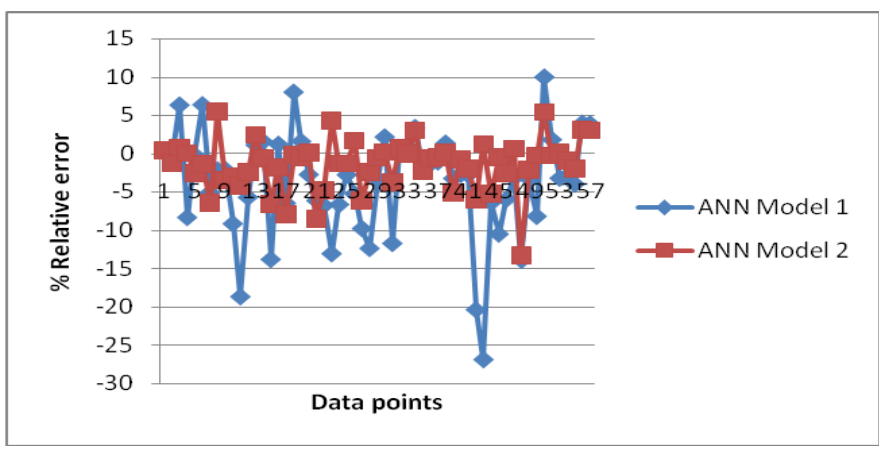

Figure 7: Comparison of the relative error for estimation of mole \% of acetic acid for ANN model $1 \& 2$ for training data set.

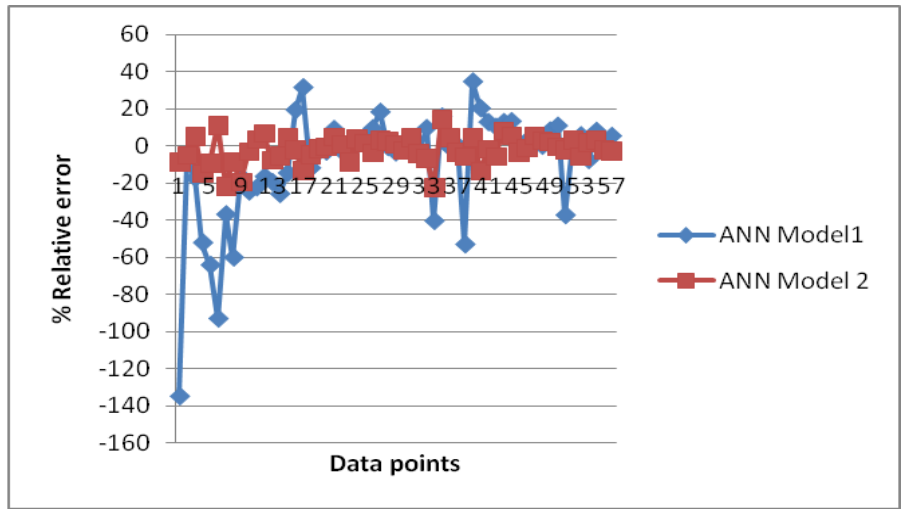

\subsubsection{Comparison of the accuracy of the Model 1 and Model 2 for test data set}

The accuracy of the ANN models developed has been further studied by calculating the relative error for all the data points of the output parameters. Fig no.8 \& 9 show the graphs plotted between the $\%$ relative error for the output parameters, composition of mixture expressed as mole \% water and composition of mixture expressed as mole \% acetic acid, for ANN model $1 \&$ ANN model 2 respectively for training data set. It is seen that there are deviations of the percentage relative errors from the mean path of the ANN model $1 \& 2$. The range of relative errors for the output parameter, composition of mixture expressed as mole $\%$ water, for ANN model 1 \& ANN model 2 is $0-10 \%$ \& $0-20 \%$ respectively. Similarly the range for the second output parameter, composition of mixture expressed as mole $\%$ acetic acid, for ANN model 1 \& ANN model 2 is $0-3 \%$ \& $0-20 \%$ respectively. As the \% relative error for ANN model 1 is lower for both the output parameters than estimated by ANN model 1 , hence it can be inferred that the ANN model 1 is superior to the ANN model 2. 
Figure 8: Comparison of the $\%$ relative error for estimation of mole \% of water for ANN model $1 \& 2$ for test data set

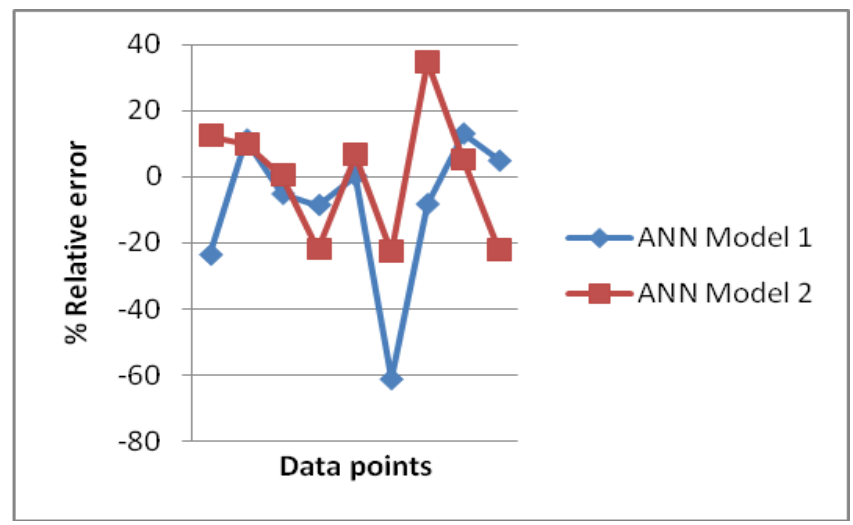

Figure 9: Comparison of the $\%$ relative error for estimation of mole \% of acetic acid for ANN model $1 \& 2$ for test data set

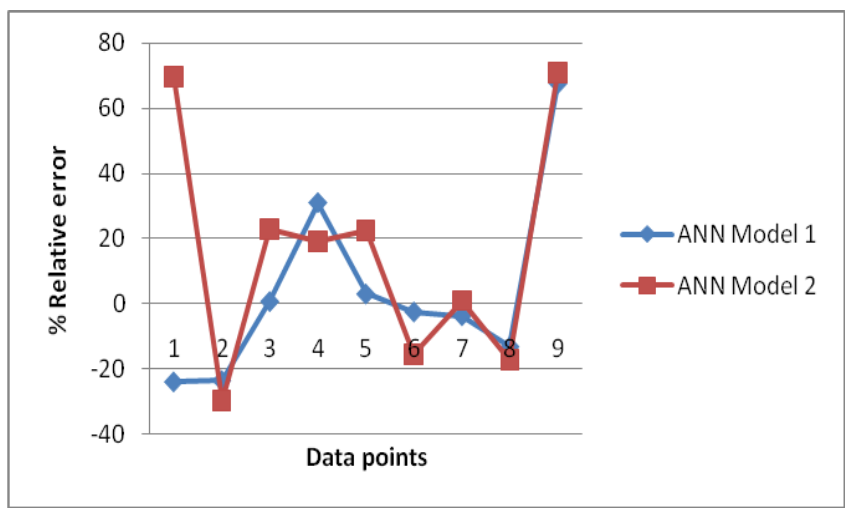

\subsection{CONCLUSION}

The present work has been successful in demonstrating the utility of artificial neural network in estimating composition of a ternary liquid mixture with its physical properties such as refractive index, $\mathrm{pH} \&$ conductivity . The ANN model 2 developed has accuracy level of $0-3 \%$ for output parameter, mole \% water \& $0-5 \%$ for output parameter, mole \% acetic acid for training data set \& ANN model 1 having accuracy level of $0-10 \%$ for output parameter, mole $\%$ water \& $0-3 \%$ for output parameter, mole $\%$ acetic acid for test data set.

It can be concluded that the present work has successfully targeted the objective. It is felt necessary that similar such case studies should be carried out to further substantiate this claim.

\section{ACKNOWLEDGEMENT}

Authors are thankful to Director, LIT, Nagpur for the facilities and encouragement provided.

\subsection{REFERENCES}

[1] Noelia Calvar, Elena Gómez , Begoña González and Ángeles DomínguezDepartamento de Ingeniería Química de la Universidad de Vigo, 36310 Vigo, SpainJ. Chem. Eng. Data, 2009, 54 (8), pp 2229-2234

[2] Jhoany Acosta-Esquijarosa, Ivonne Rodríguez-Donis Eladio Pardillo-Fontdevila Centro de Química Farmacéutica, 200 y 21, Atabey, Apdo 16042, Playa, Ciudad de la Thermochimica Acta Volume 443, Issue 1, 1 April 2006, Pages 93-97

[3] Anderson J.A, An Introduction to Neural Networks (Prentice-Hall of India, Pvt Ltd New Delhi), 1999.

[4] Rumelhart D E \& McClleland Back Propagation Training Algorithm Processing, M.I.T Press, Cambridge Massachusetts, (1986).

[5] Fan J Y, Nikolau M \& White R E, AIChE, 39 (1) (1993) 82.

[6] Hoskins J C, Kaliyur K M \& Himmelblau D M, AIChE, 37(1) (1991) 137.

[7] Watanabe K, Abe M, Kubota M \& Himmelblau D M, AIChE, 35 (11) (1989) 1803.

[8] Belsito,S., Lombardi,P., Andreussi,P., Banerjee,S. AIChE 44 (12), (1998), 2675.

[9] Pandharipande S L \& Badhe Y P, Chem Eng World 38 (6) (2003) 70 .

[10] Zamankhan P, Malinen P \& Lepomaki H, AIChE, 43 (7), (1997) 1684

[11] Baratti R, Vacca G \& Servida A, Hydrocarbon Processing, (1995) 35.

[12] Pandharipande S L, Agarwal R S, Gogte B B \& Badhe Y P, Chem Eng World 38 (5) (2003) 78.

[13] Pandharipande S L \& Badhe Y P, Chem Eng World 38 (8) (2003) 82

[14] Pandharipande S L \& Badhe Y P IIChe, 45 (4) (2003) 256.

[15] Pandharipande S L \& Mandavgane S A, Indian J Chem Tech, 11 (6) (2004) (820).

[16] Pandharipande S L, Bhaise A \& Poharkar A, Chem Eng world, 39 (1) (2004) 50

[17] Pandharipande S L \& Badhe Y P, J Inst Eng, 84 (3) (2004) 65

[18] S. A. Mehlman, P. D. Wentzell, V. L. McGuffin, Analytica Chimica Acta, 371, (1998) 117-130.

[19] Pandharipande S L \& Badhe $\mathrm{Y}$ P, elite-ANN ${ }^{\odot}$, ROC No SW-1471/2004. 